\title{
TGF- $\beta 1$-mediated transition of resident fibroblasts to cancer-associated fibroblasts promotes cancer metastasis in gastrointestinal stromal tumor
}

Hyunho Yoon ${ }^{1,2}$, Chih-Min Tang ${ }^{1,2}$, Sudeep Banerjee ${ }^{1,2,3}$, Antonio L. Delgado ${ }^{1,2}$, Mayra Yebra ${ }^{1,2}$, Jacob Davis ${ }^{1,2}$ and Jason K. Sicklick $\mathbb{B}^{1,2}$

\begin{abstract}
Cancer-associated fibroblasts (CAFs) are the most abundant cells in the tumor microenvironment. Crosstalk between tumor cells and CAFs contributes to tumor survival in most epithelial cancers. Recently, utilizing gastrointestinal stromal tumor (GIST) as a model for sarcomas, we identified paracrine networks by which CAFs promote tumor progression and metastasis. However, the mechanisms by which CAFs arise in sarcomas remain unclear. Here, RNA sequencing analysis revealed that transforming growth factor- $\beta 1$ (TGF- $\beta 1$ ) is highly expressed in both tumor cells and CAFs. To determine the functional role of TGF- $\beta 1$, we treated normal gastric fibroblasts (GFs) with recombinant TGF- $\beta 1$, which caused the GFs to adopt a more stellate morphology, as well as increased the mRNA expression of CAFmediated genes (CCL2, RAB3B, and TNC) and genes encoding fibroblast growth factors (FGFs). Moreover, while either GIST or CAF conditioned media enhanced the transition from GFs to CAFs, a TGF- $\beta 1$-blocking antibody attenuated this effect. Transwell migration assays revealed that the TGF- $\beta 1$-mediated transition from GFs to CAFs enhanced tumor cell migration. This migratory effect was abrogated by an anti-TGF- $\beta 1$ antibody, suggesting that TGF- $\beta 1$ secreted from GIST cells or CAFs is associated with GIST migration via GF-to-CAF transition. In addition, the murine spleen-to-liver metastasis model showed that GF pre-treated with TGF- $\beta 1$ promoted GIST metastasis. Collectively, these findings reveal unappreciated crosstalk among tumor cells, CAFs, and normal resident fibroblasts in the stroma of sarcomas, which enhances a GF-to-CAF transition associated with tumor migration and metastasis.
\end{abstract}

\section{Introduction}

A gastrointestinal stromal tumor (GIST), the most common sarcoma, is most often driven by oncogenic KIT or PDGFRA mutations ${ }^{1-3}$. As a result, anti-KIT/PDGFRA tyrosine kinase inhibitors (TKIs) are commonly used to treat these tumors. However, $50 \%$ of metastatic GISTs treated with imatinib, the first-line treatment, will develop drug-resistance within 20 months of starting therapy [median progression-free survival (PFS) 20.4 month] ${ }^{4}$. The objective response rates for later line anti-GIST TKIs,

\footnotetext{
Correspondence: Jason K. Sicklick (jsicklick@health.ucsd.edu)

'Department of Surgery, Division of Surgical Oncology, University of California, San Diego, CA, USA

${ }^{2}$ Moores Cancer Center, University of California, San Diego, CA, USA

Full list of author information is available at the end of the article
}

sunitinib, regorafenib, and ripretinib are only $7.0 \%, 4.5 \%$, and $9.4 \%$, respectively ${ }^{5-7}$. Due to the high rates of recurrence, alternative therapeutic targets are needed to effectively treat GIST.

Cancer-associated fibroblasts (CAFs) are the most abundant cells in the tumor microenvironment (TME). It is well established that crosstalk between epithelial cancers and CAFs can promote tumor progression and chemoresistance $^{8-10}$. Specifically, CAFs can promote a favorable biological environment for tumor growth by secreting cytokines, chemokines, and growth factors. Over time, the spatial and temporal interactions of tumor cells and stromal cells create a symbiotic relationship in which the tumor receives a growth advantage from the CAFs, and vice versa ${ }^{11}$. In addition, our previous study showed

\section{(c) The Author(s) 2021}

(c) Open Access This article is licensed under a Creative Commons Attribution 4.0 International License, which permits use, sharing, adaptation, distribution and reproduction c. in any medium or format, as long as you give appropriate credit to the original author(s) and the source, provide a link to the Creative Commons license, and indicate if changes were made. The images or other third party material in this article are included in the article's Creative Commons license, unless indicated otherwise in a credit line to the material. If material is not included in the article's Creative Commons license and your intended use is not permitted by statutory regulation or exceeds the permitted use, you will need to obtain permission directly from the copyright holder. To view a copy of this license, visit http://creativecommons.org/licenses/by/4.0/. 
paracrine fibroblastic support in GIST promotes tumor growth and metastasis ${ }^{12}$. These studies suggest that CAFs might be an alternative target for treating both epithelial cancers and sarcomas.

CAFs originate from various cell types, including resident fibroblasts, epithelial cells, and bone marrow-derived mesenchymal stem cells. Cytokines, chemokines, and growth factors can enhance their transition into CAFs in tumor stroma ${ }^{13-15}$. Transforming growth factor- $\beta 1$ (TGF$\beta 1$, a ligand of the TGF- $\beta$ receptor complex, plays important roles in many biological processes by activating TGF- $\beta$ signaling, which promotes tumorigenesis and metastasis ${ }^{16-18}$. Moreover, normal fibroblasts derived from bone marrow-derived mesenchymal stem cells in pancreatic cancer stroma have been reported to transition into CAFs in response to TGF- $\beta$ signaling, which enhances tumor growth and invasion ${ }^{13}$. However, little is known regarding the role of TGF- $\beta$ signaling in the stroma of sarcomas.

Here, we present a detailed mechanism for the transition of gastric fibroblasts (GFs) into CAFs in GIST, and how this transition promotes tumor migration and metastasis. First, TGF- $\beta 1$ secretion from GISTs and CAFs enhances a transition from normal resident fibroblast to CAFs. In turn, these ligand-dependent events lead to increased tumor motility. Overall, this study suggests that the TGF- $\beta 1$-mediated GF-to-CAF transition in the TME plays an important role in sarcoma migration and metastasis, implying that this paracrine network might be a potential target for treating sarcomas.

\section{Results}

\section{CAFs and GIST cells induce a GF-to-CAF transition}

Recently, we discovered that there are CAFs in GIST (Supplementary Fig. S1a), and platelet-derived growth factor C (PDGFC) secreted from CAFs promotes tumor progression and metastasis via a paracrine PDGFCPDGFRA-SLUG axis $^{12}$. Since the CAFs were isolated from gastric GIST, we obtained a commercially available normal GF line to compare with CAFs. To understand the differences between resident GFs and CAFs isolated from human GIST, we compared the morphology and mRNA expression of $P D G F C$, as a functional biomarker of CAFs, in these cells. We observed that CAFs had a more stellate morphology and higher expression of $P D G F C$ as compared to GFs (Fig. 1a, b). In addition, CAFs from GISTs with different mutations (i.e., KIT or PDGFRA) and distinct locations (i.e., stomach and rectum) were isolated and characterized. The quantitative polymerase chain reaction (qPCR) analysis showed that PDGFC mRNA was highly expressed in all CAF lines (Supplementary Fig. S1b).

We then cultured the GFs with either GIST-T1 or CAF conditioned media $(\mathrm{CM})$. Both $\mathrm{CM}$ increased mRNA expression of PDGFC in GFs (Fig. 1c). In addition, we compared mRNA expression of CAF-mediated genes (CCL2, RAB3B, and TNC) in GFs and CAFs. CCL2, $R A B 3 B$, and $T N C$ have known CAF markers in gene set enrichment analyses (GSEA; MISHRA_CAF_UP) ${ }^{19}$. The expression levels of $C C L 2, R A B 3 B$, and $T N C$ were also increased in CAFs compared to GFs (Fig. 1d; Supplementary Fig. S1c). CM from GIST-T1 cells and CAFs resulted in increased mRNA expression of CCL2, RAB3B, and TNC in GFs (Fig. 1e), suggesting that CM of GIST and CAFs can promote a GF-to-CAF transition.

To further investigate the functional role of PDGFC secreted from CAFs in GIST, a phosphokinase array was probed to compare CM from CAFscramble (scr), CAFshPDGFC \#1, and CAFshPDGFC \#2 in GIST-T1. Notably, CAFscr CM significantly activated several phosphokinase proteins, including JNK1/2/3, AKT, FAK, PRAS40, c-Jun, and STAT3, while these effects were abrogated by loss of PDGFC (Fig. 1f, g). In addition, as shown in supplementary Fig. S1d, CAFs markedly induced phosphorylation of PDGFRA and PDGFRB, while this effect was decreased by the loss of PDGFC. Because PDGFC is not known to activate PDGFR- $\beta / \beta$, these results suggested that CAF-mediated PDGFC secretion affects both PDGFR- $\alpha / \alpha$ homo-dimerization and PDGFR$\alpha / \beta$ hetero-dimerization, supporting our previous finding that CAF-mediated PDGFC secretion enhances GIST progression and metastasis. Together, these data demonstrated that PDGFC could be a CAF marker that modulates an aggressive phenotype of GIST cells.

\section{FGFs are overexpressed in CAFs}

Next, we performed RNA sequencing analyses (RNAseq) using GIST-T1 cells and CAFs to characterize the CAFs (GSE143547). Interestingly, RNA-seq revealed that genes encoding various fibroblast growth factors (FGFs) were overexpressed in CAFs (Fig. 2a). These genes are known to be upregulated in many cancers, including prostate, breast, and colon cancer, as well as are associated with poor survival by promoting tumor growth, metastasis, and angiogenesis ${ }^{20-22}$. Thus, we next evaluated the expression of these FGF genes by qPCR. Among them, CAFs expressed more FGF1, FGF5, and FGF9 expression than GFs (Fig. 2b). In addition, these FGFencoding genes were significantly increased in GFs with the application of CM from either GIST-T1 cells or CAFs (Fig. 2c), indicating that the expression of these genes might influence the GIST phenotype.

\section{TGF- $\beta 1$ is highly expressed in GISTs and CAFs}

TGF- $\beta$ signaling contributes to various biological processes that promote tumorigenesis and metastasis ${ }^{16,17}$. In pancreatic cancer stroma, for example, normal fibroblasts convert into CAFs via TGF- $\beta$ signaling, and this process is associated with tumor growth and invasion ${ }^{13}$. Our RNA-seq 


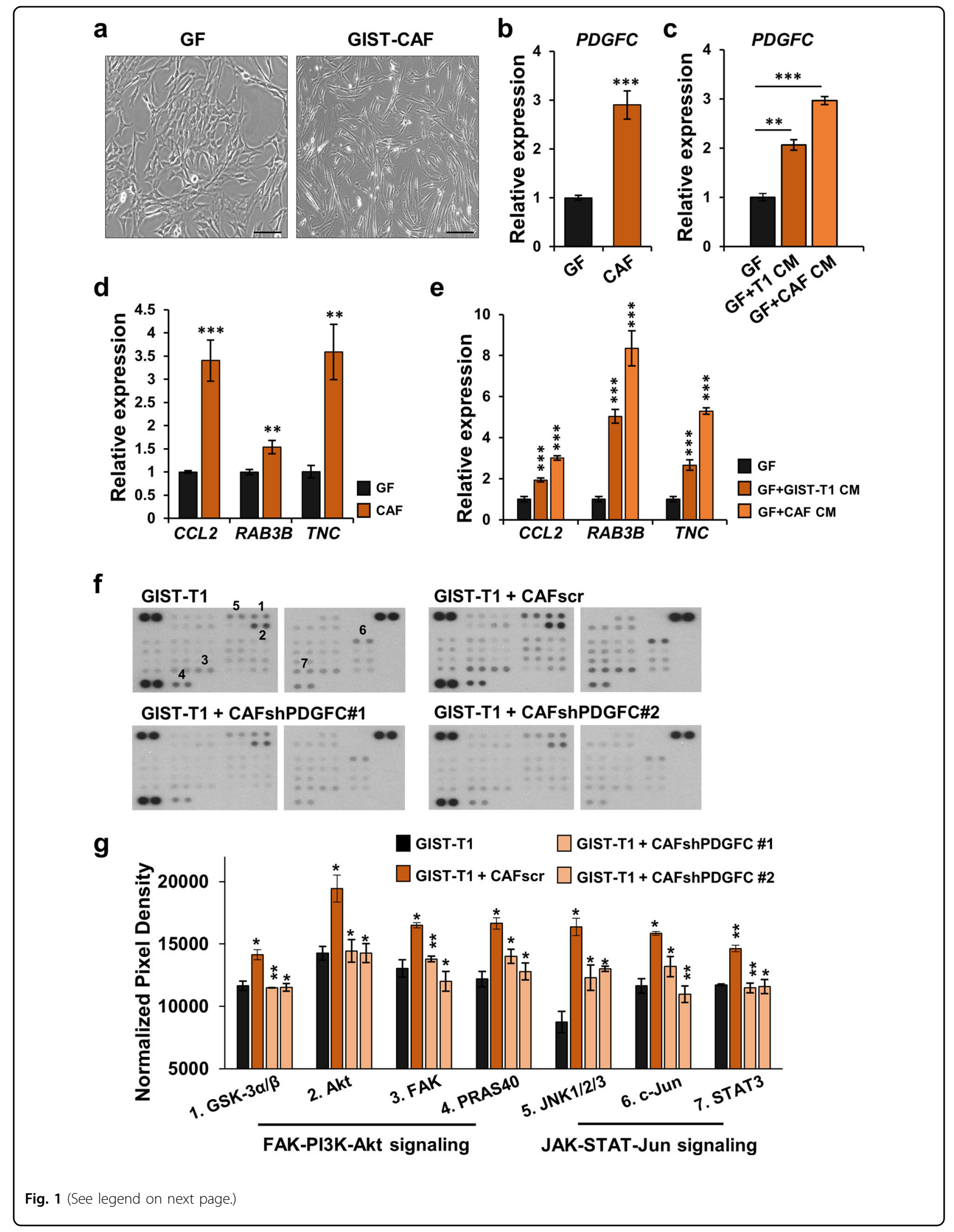


(see figure on previous page)

Fig. 1 GIST cells and CAFs enhance a GF-to-CAF transition. a The images of gastric fibroblasts (GFs) and GIST-CAFs. Scale bars, $50 \mu \mathrm{m}$. b Expression of PDGFC mRNA in GFs and CAFs by quantitative RT-PCR (qPCR). c The effects of GIST-T1 conditioned media (CM) and CAF CM treatment on PDGFC expression in GFs. After GFs were treated with CM of GIST-T1 and CAFs for $48 \mathrm{~h}$, the expression level of PDGFC was determined by qPCR. $\mathbf{d}$ Expression of CAF markers (CCL2, RAB3B, and TNC) in GFs and CAFs by qPCR. e The effects of GIST-T1 CM and CAF CM treatment on CAF markers expression in GFs. All graphs show mean \pm SEM, and $p$ values were represented by Student's $t$ test or ANOVA analysis. ${ }^{* *} p<0.01$, ${ }^{* * *} p<0.001$. f Phosphokinase array in GIST-T1 treated with CAFscr CM, CAFshPDGFC \#1 CM, or CAFshPDGFC \#2 CM. $\mathbf{g}$ The average signal (pixel density) was normalized with positive control and analyzed among samples. All graphs show mean $\pm \mathrm{SEM}$, and $p$ values were represented by ANOVA analysis. ${ }^{*} p<0.05 ;{ }^{* *} p<0.01$.
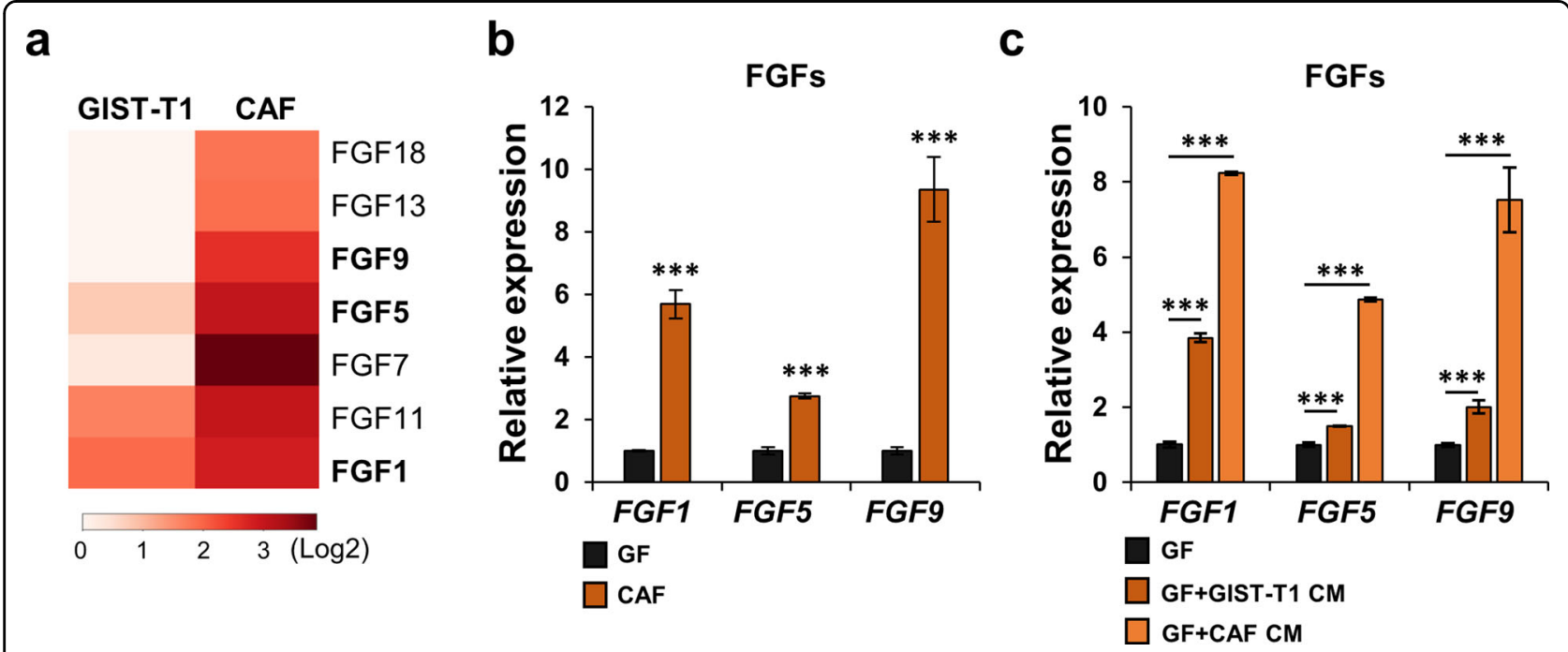

Fig. 2 FGFs are overexpressed in CAFs. a Heatmap of the expression levels of FGF genes in GIST-T1 and CAFs analyzed from RNA-seq (GSE143547). b Expression of FGF1, FGF5, and FGF9 in GFs and CAFs by qPCR. c GIST-T1 CM and CAF CM enhanced the expression of FGF1, FGF5, and FGF9 in GF. The level of expression was evaluated by qPCR. All graphs show mean \pm SEM, and $p$ values were represented by Student's $t$ test or ANOVA analysis. ${ }^{* *} p<0.01,{ }^{* * *} p<0.001$.

data also revealed that TGFB1 was expressed in both GIST-T1 (transcripts per kilobase million; TPM, 724) and CAFs (TPM, 4548). Given this observation, we performed immunohistochemical (IHC) and immunofluorescent (IF) staining using human GIST frozen sections with an anti-TGF- $\beta 1$ antibody. Both staining procedures demonstrated that TGF- $\beta 1$ was highly expressed in resected human GIST (Fig. 3a, b; Supplementary Fig. S2a, b). Next, to determine the levels of TGFB 1 mRNA and TGF- $\beta 1$ secretion, we performed qPCR and ELISA in GFs, CAFs, and GISTs. These data showed that both mRNA and protein levels of TGF- $\beta 1$ were overexpressed in GIST cultures and CAFs compared to GFs (Fig. 3c, d). In addition, to test whether TKI treatment influences the secretion of TGF- $\beta 1$, we performed qPCR of TGFB1 in GIST-T1 treated with imatinib and sunitinib. Neither imatinib nor sunitinib treatment influenced TGFB1 mRNA expression (Supplementary Fig. S2c), suggesting that tyrosine kinasemediated signaling in GIST cells did not affect TGF- $\beta 1$ expression.
TGF- $\beta 1$ promotes a GF-to-CAF transition, which increases GIST migration

We next explored whether TGF- $\beta 1$ is sufficient for the transition of resident GFs to CAFs. Given that both GIST$\mathrm{T} 1$ and CAFs overexpressed TGF- $\beta 1$, we investigated the effects of this growth factor on GFs. Human recombinant TGF- $\beta 1(10 \mathrm{ng} / \mathrm{mL})$ treatment of GFs was associated with the development of a more stellate morphology and increased mRNA expression of CCL2, RAB3B, and TNC (Fig. 4a, b). TGF- $\beta 1$ treatment also increased mRNA expression of PDGFC, FGF1, FGF5, and FGF9. However, the elevated expression of these TGF- $\beta 1$-mediated $F G F$ genes and PDGFC was abrogated by an anti-TGF- $\beta 1$ blocking antibody (Fig. 4c; Supplementary Fig. S3a, b). Interestingly, GFs stimulated by TGF- $\beta 1$ significantly increased mRNA expression of TGFB1 in GFs (Supplementary Fig. S3c), supporting the notion of autocrine and paracrine signaling, leading to transdifferentiation from GFs to CAFs. Moreover, Transwell migration assays showed that GFs treated with human recombinant TGF$\beta 1(10 \mathrm{ng} / \mathrm{mL})$ strongly enhanced migration of GIST-T1 


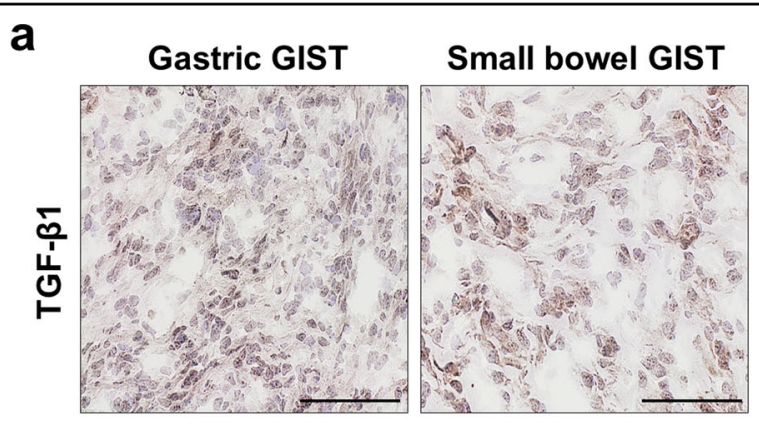

b
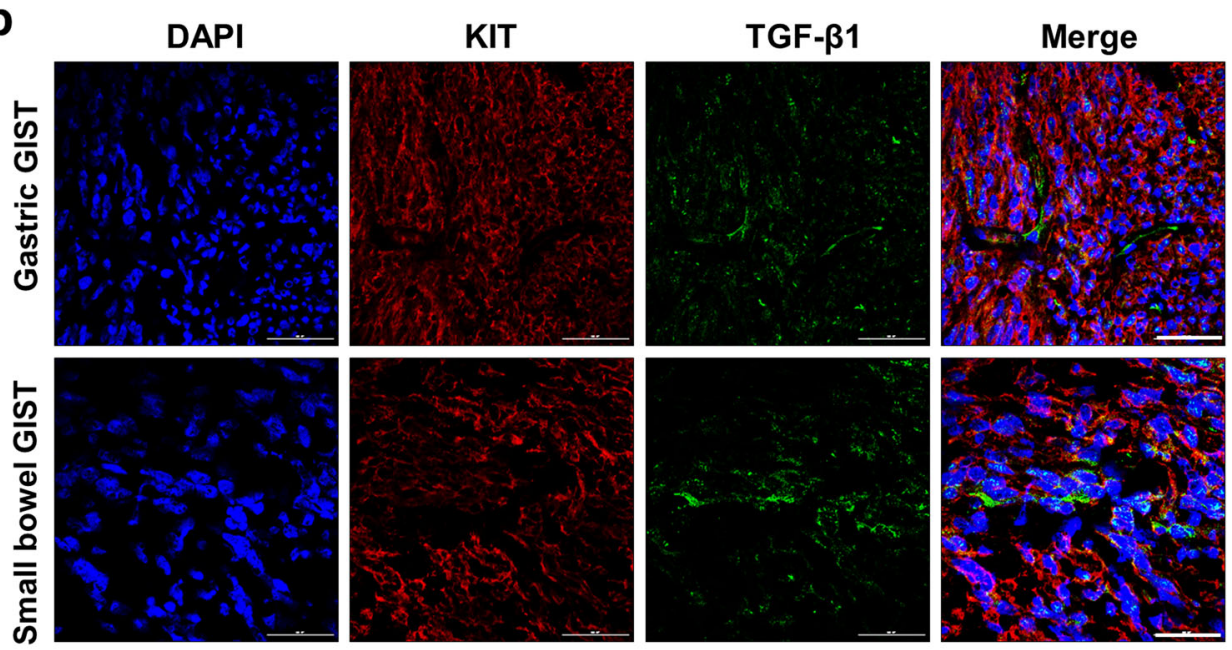

$$
\text { - DAPI }- \text { KIT }=\text { TGF- } \beta 1
$$
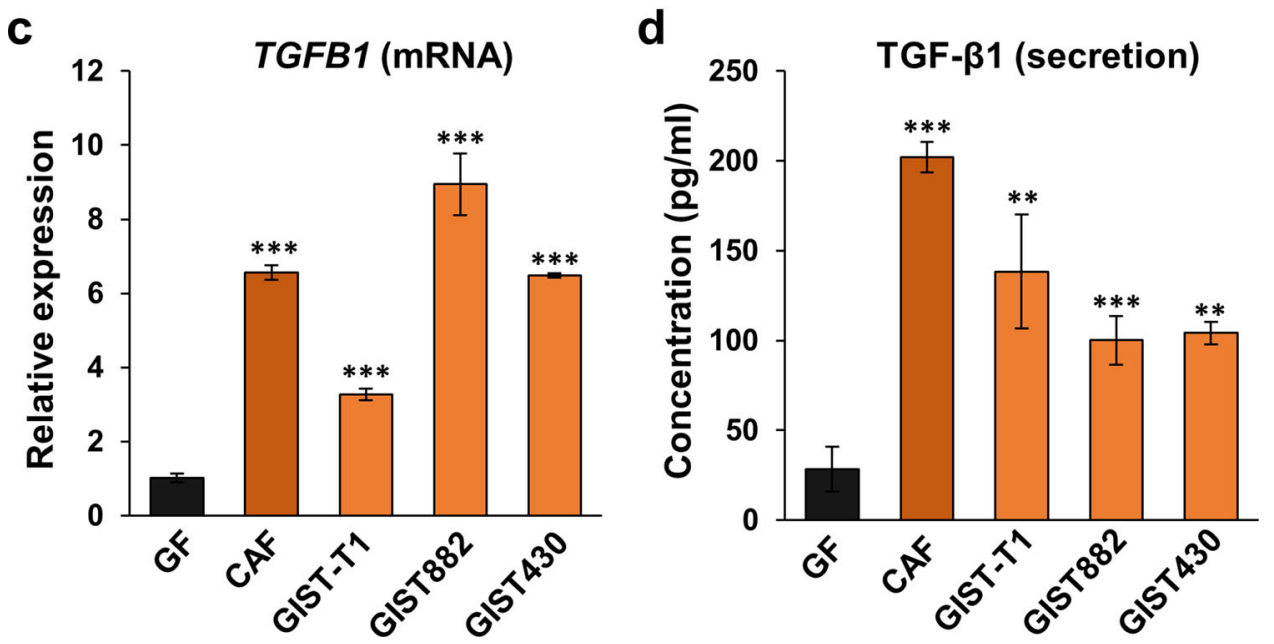

Fig. 3 TGF- $\beta 1$ is highly expressed in GIST cells and CAFs. a Representative immunohistochemistry (IHC) images with staining for TGF- $\beta 1$ in the frozen tumor sections collected from human GISTs harboring mutant KIT. Scale bars, $100 \mu \mathrm{m}$. b Representative immunofluorescence (IF) images of TGF- $\beta 1$ (green), KIT (GIST marker; red), and DAPI (nuclei; blue) staining in the resected sections. Scale bars, $50 \mu \mathrm{m}$. c mRNA expression of TGFB1 in GFs, GIST-T1, GIST882, GIST430, and CAFs by qPCR. d Comparison of TGF- $\beta 1$ levels measured by enzyme-linked immunosorbent assay (ELISA) in GFs, GISTT1, GIST882, GIST430, and CAFs. After each cell line was seeded for $48 \mathrm{~h}$ in the absence of FBS, the supernatant was collected to perform ELISA. All graphs show mean $\pm \mathrm{SEM}$, and $p$ values were represented by ANOVA analysis. ${ }^{* *} p<0.01,{ }^{* * *} p<0.001$.

and GIST882 cells, while this effect was significantly decreased by an anti-TGF- $\beta 1$ blocking antibody (Fig. $4 \mathrm{~d}-\mathrm{g}$ ). To test whether an isotype control for the
TGF- $\beta 1$ blocking antibody influences GIST migration, we performed Transwell migration assays with an isotype control antibody (mouse IgG) in GIST cells. This did not 

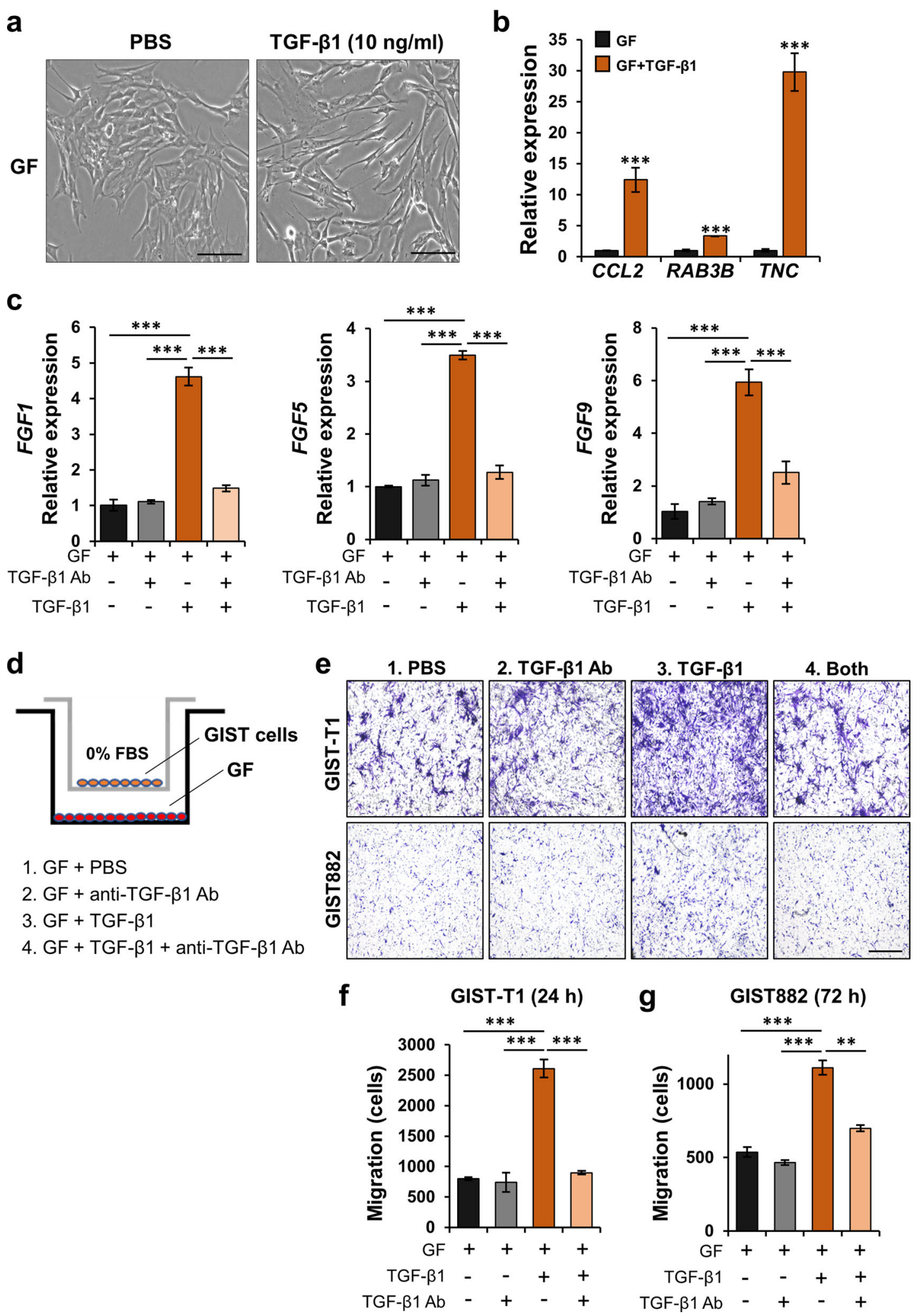

Fig. 4 (See legend on next page.) 
(see figure on previous page)

Fig. 4 TGF- $\beta 1$ is associated with a GF-to-CAF transition, which increases GIST migration. a Photographic images of GFs treated with human recombinant TGF- $\beta 1$. Scale bars, $50 \mu \mathrm{m}$. GFs were treated with TGF- $\beta 1(10 \mathrm{ng} / \mathrm{mL})$ for $48 \mathrm{~h}$. $\mathbf{b}$ Effect of TGF- $\beta 1$ treatment on mRNA expression of CAF markers (CCL2, RAB3B, and TNC) in GFs by qPCR. c Effect of anti-TGF- $\beta 1$ blocking antibody on the expression of FGF1, FGF5, and FGF9 in GFs treated with TGF- $\beta 1$. GFs were treated with TGF- $\beta 1(10 \mathrm{ng} / \mathrm{mL})$ and/or anti-TGF- $\beta 1$ blocking antibody $(1 \mu \mathrm{g} / \mathrm{mL})$ for $48 \mathrm{~h}$. The expression levels of these genes were measured by qPCR. $\mathbf{d}$ Experimental design for Transwell migration assay of anti-TGF- $\beta 1$ blocking antibody. After GFs were seeded on the bottom, TGF- $\beta 1(10 \mathrm{ng} / \mathrm{mL})$ and anti-TGF- $\beta 1$ blocking antibody $(1 \mathrm{\mu g} / \mathrm{mL})$ were added for $24 \mathrm{~h}$. e-g Representative images (e) of the migrated GIST cells stained with $0.05 \%$ crystal violet in the indicated group and quantitative data in GIST-T1 cells (f) and GIST882 cells (g) analyzed by ImageJ software. Scale bars, $200 \mu \mathrm{m}$. All graphs show mean \pm SEM, and $p$ values were represented by Student's $t$ test or ANOVA analysis. ${ }^{* *} p<0.01$, ${ }^{* * *} p<0.001$.

affect tumor cell migration (Supplementary Fig. S3d). Together, these data indicated that TGF- $\beta 1$ is involved in GF-to-CAF transition, which leads to increased GIST migration.

\section{TGF- $\beta 1$ secretion from GIST cells or CAFs enhances cell migration}

To investigate the effect of TGF- $\beta 1$ secreted from GIST cells or CAFs, we performed Transwell migration assays with CAF CM, GIST CM, and anti-TGF- $\beta 1$ blocking antibody (Fig. 5a). The migration assays showed that GFs with CM from CAFs strongly enhanced GISTT1 cell migration compared to GF only, while the effect was markedly decreased by anti-TGF- $\beta 1$ blocking antibody (Fig. 5b, c). Moreover, given that TGF- $\beta 1$ was highly expressed in GIST lines, we performed Transwell migration assays with CM of GIST cell lines. The assays showed that CM of GISTs (GIST-T1, GIST882, and GIST430) in GFs increased GIST-T1 migration, while this effect was abrogated by anti-TGF- $\beta 1$ antibody (Fig. $5 \mathrm{~d}$, e). These data suggested that TGF- $\beta 1$ secreted from CAFs and various GIST lines with different KIT mutations can enhance a resident GF-to-CAF transition, which leads to increased GIST cell migration.

\section{The TGF- $\beta 1$-mediated transition of GF to CAF promotes GIST metastasis in vivo}

We next examined the metastatic effect of TGF- $\beta 1$ mediated transition from GF to CAF using our murine spleen-to-liver metastasis model. The spleens of nude mice ( $n=5$ per group) were injected with a green fluorescent protein (GFP)-labeled GIST-T1, GFP-labeled $\mathrm{T} 1+\mathrm{GFs}$, and GFP-labeled T1 + GFs pretreated with TGF- $\beta 1$. The mixture of GIST-T1 cells with GFs pretreated with TGF- $\beta 1$ increased tumor formation at the injection site and liver metastases (Fig. 6a-d; Supplementary Fig. S4a, b). H\&E staining and IHC with anti-KIT antibody using formalin-fixed paraffin-embedded (FFPE) liver sections from each group also supported that TGFB1-mediated GF to CAF transdifferentiation increased GIST liver metastases (Fig. 6e; Supplementary Fig. S4c). IHC analysis in the same sections did not reveal FSP-1positive cells in the liver, suggesting that only GIST cells stimulated (i.e., preconditioned) by CAFs (or stimulated GFs) metastasized to the liver (data are now shown). Together, these data demonstrated that TGF- $\beta 1$ secretion from CAFs and GISTs enhances a GF-to-CAF transition, which promotes GIST migration and metastasis via paracrine signaling (Fig. 6f).

\section{Discussion}

Cancer cells are in constant communication with various cells in the tumor stroma. CAFs are one of the major components in the TME, and crosstalk between cancer cells and CAFs contributes to tumor growth, invasion, and metastasis, as well as drug resistance ${ }^{23-25}$. In our previous study, we showed for the first time that CAFs isolated from resected human GIST play important roles in GIST progression and metastasis, suggesting the concept that targeting CAFs may represent a novel therapeutic target for treating sarcomas.

CAFs have been studied extensively and the processes of CAF development are generally known in many carcinomas. For instance, hypoxia-inducible factor- $1 \alpha$ (HIF-1 $\alpha$ ) and CXCL12 induced by oxidative stress have been shown to enhance the transition of resident fibroblasts to CAFs in breast cancers ${ }^{26}$. In melanoma, normal endothelial cells can differentiate into activated fibroblasts via TGF- $\beta 1$-induced endothelial-to-mesenchymal transition ${ }^{27}$, which then promotes cancer progression. These studies suggested that CAFs can originate from various cell types through chemokines, cytokines, and growth factors secreted from tumor stroma. In contrast, very little is known about CAF origins in sarcomas. But, given the critical role of CAFs in GIST as a sarcoma model, we next addressed how these CAFs are derived in sarcomas. Evidence for the mechanism of CAF development in the sarcoma biology was obtained from our RNA-seq data that revealed overexpression of TGFB1 in both GIST cells and CAFs. Using resected GIST tissues, we confirmed high levels of TGF- $\beta 1$ expression by IHC and IF staining. TGF- $\beta 1$ is the most well-known isoform of the TGF- $\beta$ subfamily, and TGF$\beta 1$ expression contributes to the development of various diseases, including cancers ${ }^{28-30}$. Given these observations, we hypothesized that TGF- $\beta 1$ secreted from 
a

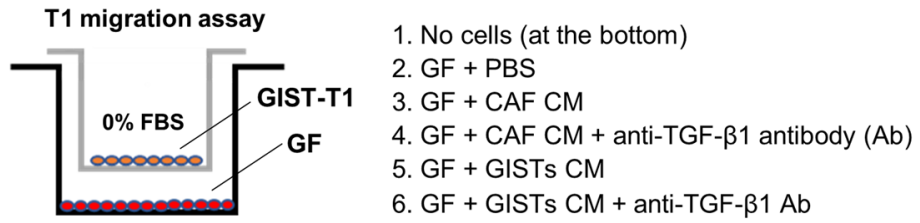

b

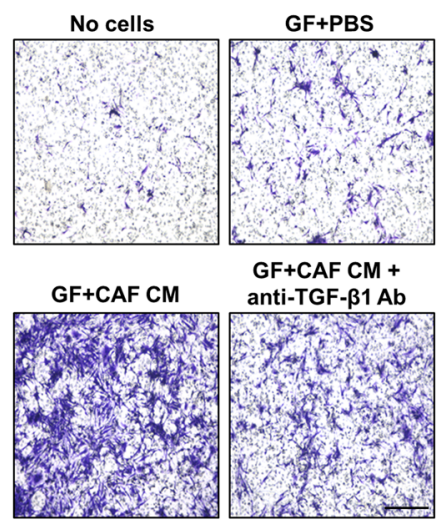

C

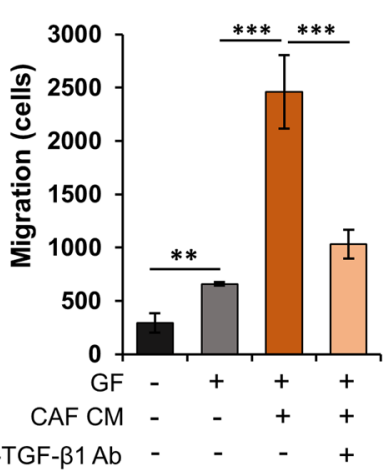

d

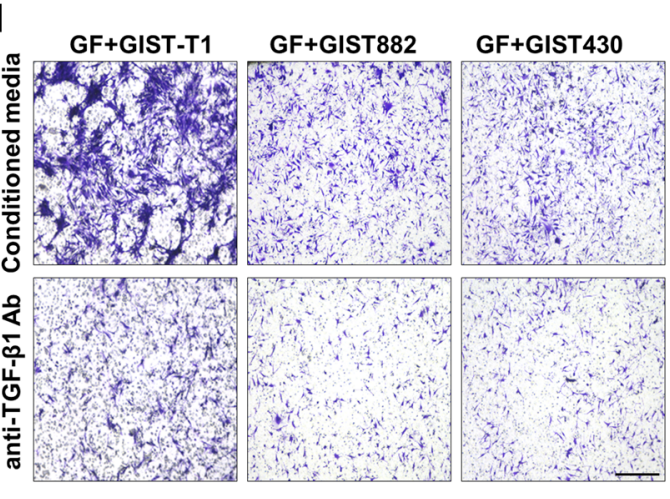

e

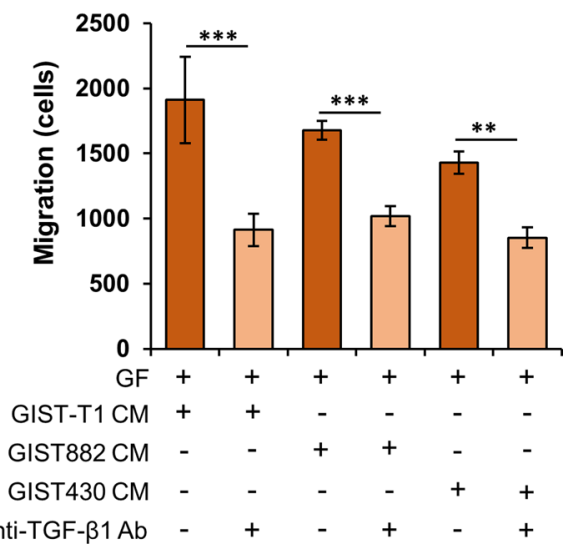

Fig. 5 TGF- $\beta 1$ secreted from GIST-T1 cells and CAFs is involved in increased GIST migration. a Experimental design of the Transwell migration assay. b, c Representative images (b) and quantitative data (c). GFs were treated with PBS (control), CAF CM, and/or TGF- $\beta 1$ blocking antibody (1 $\mu \mathrm{g} /$ $\mathrm{mL}$ ) for $24 \mathrm{~h}$ in the bottom well. The migrated GIST-T1 cells were imaged using a BZ-X800 Life Science Microscope and analyzed by ImageJ software. d Representative images showing Transwell migration assay of TGF- $\beta 1$ blocking antibody in GFs treated with CM of GIST-T1, GIST882, and GIST430. e Quantitative data of the migrated GIST-T1 cells in the indicated conditions. Scale bars, $200 \mu \mathrm{m}$. Graphs show mean \pm SEM, and $p$ values were represented by ANOVA analysis. ${ }^{* *} p<0.01,{ }^{* * *} p<0.001$.

tumor cells and CAFs is a key mediator of the transition of normal resident GFs to CAFs. Indeed, we observed that TGF- $\beta 1$ influenced the morphology and gene expression of GFs. Specifically, treatment with GIST and CAF CM caused resident GFs to adopt a more stellate morphology, and induced the expression of CAFmediated genes CCL2, RAB3B, and TNC (GSEA; MISHRA_CAF_UP $)^{19}$, while anti-TGF- $\beta 1$ blocking antibody treatment inhibited this effect, suggesting that TGF$\beta 1$ secreted from tumor cells and CAFs plays an important role for a transition from resident fibroblasts to CAFs.
In addition, we found that GFs stimulated by TGF$\beta 1$ significantly increased the mRNA expression of TGFB1 in GFs. Given the observation, we performed a Tanswell migration assay with TGF- $\beta 1$ neutralizing antibody in the stimulated GFs since TGF- $\beta 1$ is well-known as a key regulator of tumor cell migration through induction of epithelial-mesenchymal transition (EMT) in various cancers $^{31}$. The data showed that TGF- $\beta 1$ neutralizing antibody in the stimulated GFs significantly decreased GIST cell migration as compared to isotype control antibody treatment (Supplementary Fig. S4d, e). These data suggested that TGF- $\beta 1$ plays an important role 


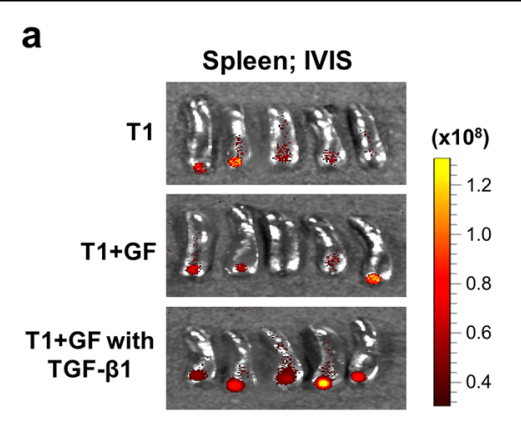

C
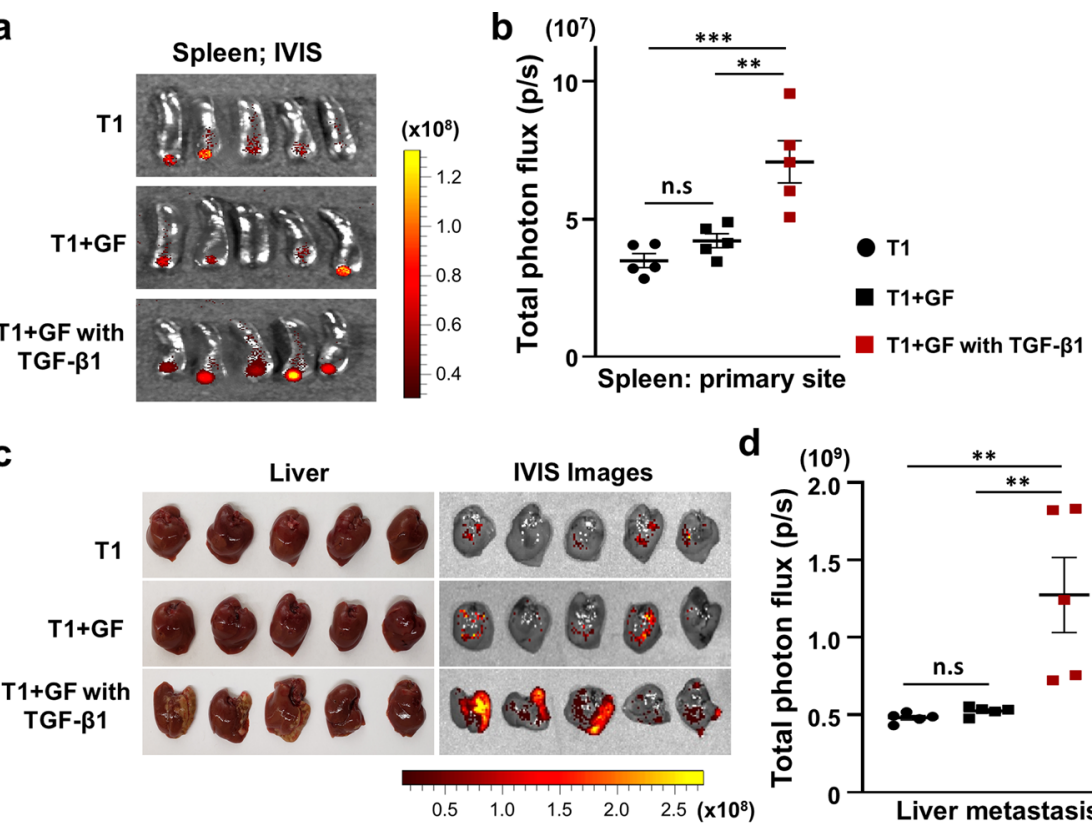

d

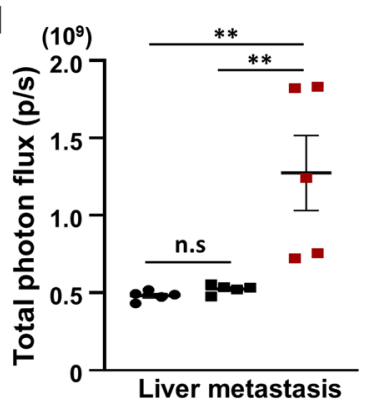

e

Liver metastasis

f
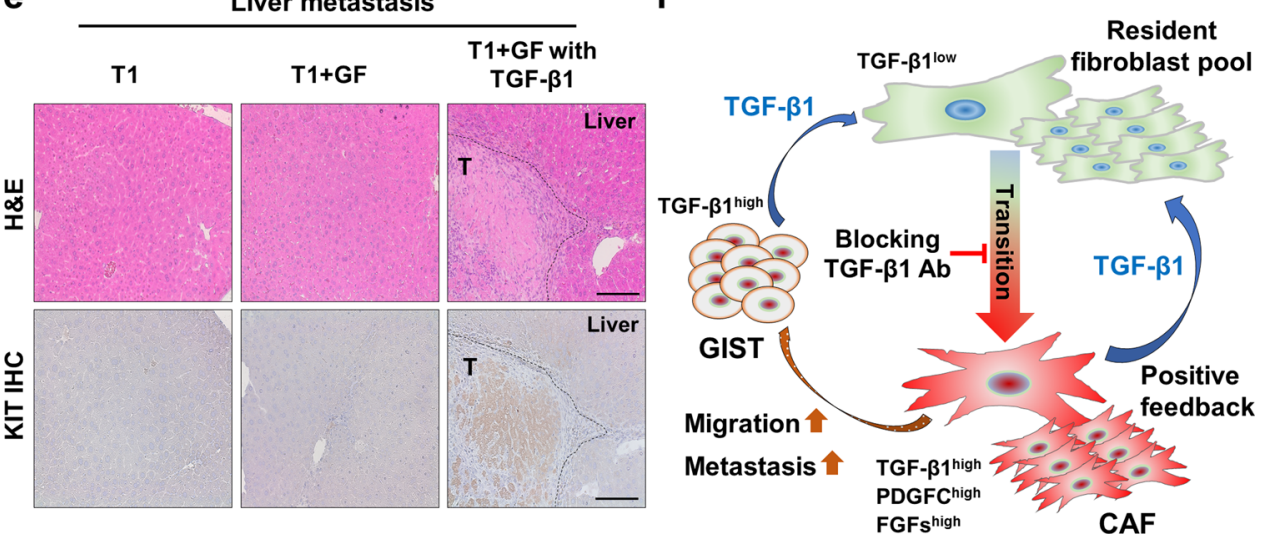

Fig. 6 TGF- $\beta$ 1-mediated transition of GF to CAF promotes GIST metastasis. $\mathbf{a}$, $\mathbf{b}$ Effects of a transition from GF to CAF on the murine spleen-toliver metastasis model. The mice were injected with GFP-labeled T1, T1 + GF, and T1 + GF pre-treated with TGF- $\beta 1$. After 3 weeks, all mice were sacrificed. The harvested spleens and livers from each mouse were analyzed using the IVIS imaging system. IVIS images (a) of the spleen and quantification (b) analyzed from total photon flux ( $p / s$ ) of the spleen. $p$ Values were represented by ANOVA analysis. ${ }^{* *} p<0.01$, ${ }^{* * *} p<0.001$. n.s. nonsignificant. c Photographic images (left) and IVIS images (right) of the liver in the indicated group. d Quantitative data were analyzed by total photon flux $(p / s)$ on the metastatic liver. $p$ Values were represented by ANOVA analysis. ${ }^{* *} p<0.01$. e Representative H\&E images (top) and IHC (bottom) stained with anti-KIT antibody in the tumor sections collected from livers. Scale bars, $100 \mu \mathrm{m}$. f Proposed model of TGF- $\beta 1$-mediated transition of GFs to CAFs in the GIST stroma. TGF- $\beta 1$ secretion from CAFs and GIST cells enhances a GF-to-CAF transition, which promotes GIST migration via paracrine signaling networks.

for GIST cell migration, as well as for GF to CAF transdifferentiation.

Our previous and present studies have shown that PDGFC could be a putative biomarker for CAFs in GIST. Elevated expression of PDGFC in CAFs promoted tumor growth and metastasis via the enhanced SLUG-mediated $\mathrm{EMT}^{12}$. Here, we provided additional evidence that the expression of FGF-encoding genes can be another putative marker of CAFs in sarcoma biology. FGF secretion is strongly associated with tumor progression and metastasis in many tumors ${ }^{20-22,32}$. The activation of GFs by TGF- $\beta 1$ led to high expression levels of several genes, including FGF1, FGF5, and FGF9. Those FGF-encoding genes may play an unappreciated role in GIST.

Herein, we demonstrated that the GFs activated by TGF- $\beta 1$ promoted GIST cell migration and metastasis in vitro and in vivo, suggesting that GIST-CAFs can originate from resident fibroblasts in response to TGF$\beta 1$ secreted by tumors and stroma. Our results open the possibility that targeting TGF- $\beta 1$-mediated paracrine 
networks might be a better strategy for sarcoma therapy. In summary, we have demonstrated the role of TGF- $\beta 1$ on the transition of GFs to CAFs, which describes a detailed mechanism of how CAFs are derived from normal resistant fibroblasts in the sarcoma microenvironment.

\section{Materials and methods Human GIST samples}

After obtaining written informed consent, GIST samples were collected from patients undergoing resections at the University of California, San Diego (UCSD). All procedures were approved by the UCSD Institutional Review Board (IRB; \#181755). Experienced pathologists made the pathological diagnosis based on light microscopic analysis of FFPE tissue sections labeled with antibodies against KIT (Santa Cruz; sc-13508) and DOG1 (Abcam; ab190721). Excess tumor tissue was used for only research purposes.

\section{Cell lines}

The human GIST cell line GIST-T1 (KIT exon 11 mutation) was from T. Taguchi (Kochi Medical School, Japan). GIST430 (KIT exon 11, 13 mutations) and GIST882 (KIT exon 13 mutation) were provided by Dr. Jonathan A. Fletcher (Dana-Farber Cancer Center, Boston, MA). Normal GFs were purchased from Science Research Laboratories (Carlsbad, CA), and CAFs were isolated from a human PDGFRA mutant GIST by a serial trypsinization method ${ }^{33}$. CAFscr, CAFshPDGFC \#1, and CAFshPDGFC \#2 cell lines were established using lentiviral transduction ${ }^{12}$. DNA authentication of CAFs was performed by short tandem repeat profiling (DDC Medical, Inc). GIST-T1, GFs, and CAFs were maintained in Dulbecco's Modified Eagle Medium (DMEM; Gibco, Grand Island, NY) with 10\% fetal bovine serum (FBS) and $1 \%$ penicillin-streptomycin (Mediatech, Manassas, VA). GIST882 was cultured in Roswell Park Memorial Institute (RPMI 1640; Gibco) with 20\% FBS and 1\% penicillin-streptomycin. GIST430 was grown in Iscove's Modified Dulbecco's Medium (IMDM; Gibco) with 15\% FBS, $2 \mathrm{mM}$ glutamine, and $1 \%$ penicillin-streptomycin. All cell lines were incubated at $37^{\circ} \mathrm{C}$ in an incubator with $5 \% \mathrm{CO}_{2}$. To monitor Mycoplasma contamination, all cell lines were regularly tested using PCR reaction with primers; forward, 5'-GGCGAATGGGTGAGTAACACG; reverse, 5'-CGGATAACGCTTGCGACCTAT.

\section{Quantitative real-time PCR (qPCR)}

RNeasy Mini Kit (Qiagen, Germantown, MD) was used to extract total RNA from GIST lines, CAFs, and GFs. cDNA was synthesized from total RNA $(1 \mu \mathrm{g})$ by First Strand cDNA Synthesis Kit (Invitrogen, Waltham, MA) according to the manufacturer's instructions. qPCR was performed using SYBR green (Bio-Rad, Hercules, CA) on the CFX96 cycler (Bio-Rad). The fold changes were normalized using GAPDH levels, and each reaction was conducted in triplicate. The primer sequences were as follows: PDGFC (forward, 5'-GGCTTCTCCTGCTGACA TCT; reverse, 5'-TCCGTTCTGTTCCTTGTTGC); CCL2 (forward, 5'-GCAGCAAGTGTCCCAAAGAA; reverse, 5'-TCGGAGTTTGGGTTTGCTTG); RAB3B (forward, 5'-AACTGCAGATCTGGGACACA; reverse, 5'-ACACT TGTTCCCCACCAGAA); TNC (forward, 5'-GAACCTG GTGTCTTCCCTGA; reverse, 5'-AGACACAGCCACAT CCTTCA); TGFB1 (forward, 5'-TACAGCAACAATTC CTGGCG; reverse, 5'-AAGCCCTCAATTTCCCCTCC); FGF1 (forward, 5'-CTGGAAAGGCTGGAGGAGAA; reverse, 5'-CGTTTGCAGCTCCCATTCTT); FGF5 (forward, 5'-AGTGGTATGTGGCCCTGAAT; reverse, 5'CTGCTCCGACTGCTTGAATC); FGF9 (forward, 5'-AT AAGCACGTGGACACTGGA; reverse, 5'-TGTGTAAT TTCTGGTGCCG); GAPDH (forward, 5'-TCGACAGTC AGCCGCATCT; reverse, 5'-TACGACCAAATCCGTTG ACTCCGA).

\section{Human phosphokinase array}

Proteome Profiler human phospho-RTK antibody arrays (ARY003; R\&D Systems, Minneapolis, MN) were tested in GIST-T1, T1 + CAFscr, T1 + CAFshPDGFC \#1,

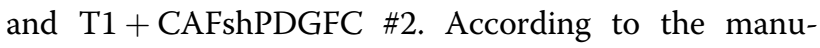
facturer's instructions, cell lysates containing a total of $500 \mu \mathrm{g}$ protein were incubated overnight with an antibody array containing 42 anti-RTK antibodies, and the bound phospho-RTKs were detected with anti-phospho-tyrosine antibody-horseradish peroxidase (HRP). Blots were normalized using the 8 anti-phosphotyrosine antibody control spots per filter using ImageJ software (RRID: SCR_003070).

\section{Western blot analysis}

GIST-T1 cells were treated with CM from CAFscr, CAFshPDGFC \#1, or CAFshPDGFC \#2 for $24 \mathrm{~h}$. The lysates with RIPA buffer (Cell Signaling Technology, Beverly, MA) were subjected to Western blot with the antibodies (1:1000 dilutions) against p-PDGFRA (Cell Signaling Technology, 4547P), PDGFRA (Cell Signaling Technology, 3164S), p-PDGFRB (ABclonal, Woburn, MA, Apo815), PDGFRB (Cell Signaling Technology, 3169 S), and $\beta$-actin (Cell Signaling Technology, 4967S). After SDS-polyacrylamide gel electrophoresis (Invitrogen) with the lysates, the gel was transferred to polyvinylidene difluoride membrane (Bio-Rad) with Trans-Blot Turbo transfer system 690BR (Bio-Rad). The immune-reactive bands with the secondary HRP-conjugated antibodies were visualized using a chemiluminescent substrate (Invitrogen) and were exposed to X-ray film (Genesee Scientific, San Diego, CA). 


\section{IHC and H\&E staining}

Resected human GIST tissues were frozen-embedded in OCT compound and sectioned at $5-\mu \mathrm{m}$ thickness. Following fixation with $4 \%$ formaldehyde, the slides were stained for TGF- $\beta 1$. Mouse liver tissues collected from the spleen-to-liver metastasis model were formalin-fixed, paraffin-embedded, and sectioned at 5- $\mu \mathrm{m}$ thickness. IHC staining was performed using $A B C$ Universal PLUS Kit (Vector Laboratories, Burlingame, CA). For antigen retrieval, the slides were boiled with IHC Antigen Retrieval Solution (Invitrogen) for $30 \mathrm{~min}$. After eliminating endogenous peroxidase activity with $0.3 \%$ hydrogen peroxide, anti-TGF- $\beta 1$ (Abcam; ab92486) and anti-KIT (Dako; A4502) antibodies were incubated overnight at $4{ }^{\circ} \mathrm{C}$ (1:250 dilution). The following day, the sections were developed with HRPconjugated secondary antibody and $\mathrm{DAB}$ chromogen provided with the kit (Vector Laboratories). For H\&E staining, the mouse liver slides were stained in hematoxylin solution (EMD Millipore) for $2 \mathrm{~min}$. After washing in running tap water for $5 \mathrm{~min}$, the slides were counterstained in eosin solution (Sigma-Aldrich) for $30 \mathrm{~s}$. The slides of IHC and H\&E were photographed using CKX53 microscopy (Olympus).

\section{IF staining}

IF staining was performed using resected tissues from two GIST patients. Briefly, the tumor sections were fixed with $4 \%$ paraformaldehyde for $10 \mathrm{~min}$. After washing the slides with PBS, the samples were blocked with $5 \%$ goat serum for $1 \mathrm{~h}$ at room temperature. The slides were then incubated with anti-c-KIT (Santa Cruz; sc-13508) and anti-TGF- $\beta 1$ antibodies (Abcam; ab92486) overnight at $4{ }^{\circ} \mathrm{C}$ (1:1000 dilutions). The signal was detected with antimouse Alexa Fluor 594 and anti-rabbit Alexa Fluor 488 (Invitrogen). Fluorescence images were visualized using a Confocal Microscope A1R (Nikon Inc.).

\section{Enzyme-linked immunosorbent assay (ELISA)}

The levels of TGF- $\beta 1$ secretion were evaluated using the TGFB1 ELISA Kit (MyBioSource, San Diego, CA). For sample preparation, cell culture supernatants were collected from GF, CAF, GIST-T1, GIST882, and GIST430 cells. After the supernatants were activated by activating reagent $(0.2 \mathrm{~N} \mathrm{HCl})$ for $10 \mathrm{~min}$, the samples were added into a 96-well microplate coated with TGF- $\beta 1$ capture antibodies. The captured TGF- $\beta 1$ was developed by Avidin-Biotin-Peroxidase complex, and the absorbance was read at $450 \mathrm{~nm}$ with a microplate reader (BioTek).

\section{Migration assay}

GFs $\left(5 \times 10^{4}\right.$ cells) were seeded with TGF- $\beta 1$ (InvivoGen, San Diego, CA), anti-TGF- $\beta 1$ blocking antibody (BioLegend, Cat\# 521707), GIST CM or CAF CM in the bottom of 24-well Transwell plates. After we washed out the media with fresh serum-free media, $5 \times 10^{4}$ GIST-T1 or GIST882 cells were seeded in the upper chamber (SARSTEDT Inc, Sparks, NV). The migrated cells adherent to the membrane under-surface were photographed using a BZ-X800 Life Science Microscope (KEYENCE, Itasca, IL). The cells were counted and analyzed by ImageJ software.

\section{Spleen-to-liver metastasis model}

The mouse experiments were conducted and approved in accordance with the Animal Care Committee of the University of UCSD (S11020). Five-week-old male Balb/c nude mice (NU/J-002019) were obtained from The Jackson Laboratory (JAX). After anesthesia, the mice were made $\sim 1 \mathrm{~cm}$ incision in the left abdominal flank. GIST-T1 (GFP-conjugated; $5 \times 10^{6}$ cells), GIST-T1 $(5 \times$ $10^{6}$ cells $)$ with GF $\left(1 \times 10^{6}\right.$ cells $)$, and GIST-T1 with GF pretreated with TGF- $\beta 1$ for $24 \mathrm{~h}$ were injected into the spleen. After 3 weeks, livers collected from each group were analyzed using the IVIS imaging system, and the GFP signals produced from livers were graphed by total photon flux $(\mathrm{p} / \mathrm{s})$.

\section{Statistical analyses}

Data are presented as the mean \pm SEM. Statistical comparisons between two groups were performed with the one-way ANOVA, followed by the Student's $t$ test. Sidak's multiple comparison test was used to compare among more than two groups. A value of $P<0.05$ was considered significant.

\section{Acknowledgements}

This work was supported in part by Biorepository and Tissue Technology Shared Resource (National Institutes of Health (NIH) grant No. P30CA023100) (J.K.S.), as well as NIH (R01 CA226803, J.K.S.), Food and Drug Administration (R01 FD006334, J.K.S.), Jonathan David Foundation (J.K.S.), GIST Research Fund (J.K.S.), and SDH Research Fund (J.K.S.).

\section{Author details}

'Department of Surgery, Division of Surgical Oncology, University of California, San Diego, CA, USA. ${ }^{2}$ Moores Cancer Center, University of California, San Diego, CA, USA. ${ }^{3}$ Department of Surgery, University of California, Los Angeles, CA, USA

Conflict of interest

J.K.S. receives research funding from Novartis Pharmaceuticals, Amgen Pharmaceuticals, and Foundation Medicine, consultant fees from Grand Rounds, Loxo, and Deciphera, speaker's fees from Roche and Deciphera, and owns stocks in Personalis.

\section{Publisher's note}

Springer Nature remains neutral with regard to jurisdictional claims in published maps and institutional affiliations.

Supplementary information The online version contains supplementary material available at https://doi.org/10.1038/s41389-021-00302-5.

Received: 11 September 2020 Revised: 17 December 2020 Accepted: 13 January 2021

Published online: 06 February 2021 


\section{References}

1. Corless, C. L. \& Heinrich, M. C. Molecular pathobiology of gastrointestinal stromal sarcomas. Annu. Rev. Pathol. 3, 557-586 (2008).

2. Corless, C. L., Barnett, C. M. \& Heinrich, M. C. Gastrointestinal stromal tumours: origin and molecular oncology. Nat. Rev. Cancer 11, 865-878 (2011).

3. Shi, E. et al. FGFR1 and NTRK3 actionable alterations in "Wild-Type" gastrointestinal stromal tumors. J. Transl. Med. 14, 339 (2016).

4. Blanke, C. D. et al. Phase III randomized, intergroup trial assessing imatinib mesylate at two dose levels in patients with unresectable or metastatic gastrointestinal stromal tumors expressing the kit receptor tyrosine kinase: S0033. J. Clin. Oncol. 26, 626-632 (2008).

5. Demetri, G. D. et al. Efficacy and safety of regorafenib for advanced gastrointestinal stromal tumours after failure of imatinib and sunitinib (GRID): an international, multicentre, randomised, placebo-controlled, phase 3 trial. Lancet 381, 295-302 (2013).

6. Demetri, G. D. et al. Efficacy and safety of sunitinib in patients with advanced gastrointestinal stromal tumour after failure of imatinib: a randomised controlled trial. Lancet 368, 1329-1338 (2006).

7. Janku, F. et al. Switch control inhibition of KIT and PDGFRA in patients with advanced gastrointestinal stromal tumor: a phase I study of ripretinib. J. Clin. Oncol. 38, 3294-3303 (2020).

8. Chen, X. \& Song, E. Turning foes to friends: targeting cancer-associated fibroblasts. Nat. Rev. Drug Discov. 18, 99-115 (2019).

9. Lenze, E. J., Rodebaugh, T. L. \& Nicol, G. E. A framework for advancing precision medicine in clinical trials for mental disorders. JAMA Psychiatry 77, 663-664 (2020).

10. Liu, T. et al. Cancer-associated fibroblasts build and secure the tumor microenvironment. Front. Cell Dev. Biol. 7, 60 (2019).

11. de la Torre, J. et al. Tumor symbiosis: gastrointestinal stromal tumor as a host for primary peritoneal mesothelioma. J. Gastrointest. Surg. 23, 879-881 (2019).

12. Yoon, $\mathrm{H}$. et al. Cancer-associated fibroblast secretion of PDGFC promotes gastrointestinal stromal tumor growth and metastasis. Oncogene (2021). https://doi.org/10.1038/s41388-021-01685-w.

13. Wen, S., Niu, Y., Yeh, S. \& Chang, C. BM-MSCs promote prostate cancer progression via the conversion of normal fibroblasts to cancer-associated fibroblasts. Int. J. Oncol. 47, 719-727 (2015).

14. Petersen, O. W. et al. The plasticity of human breast carcinoma cells is more than epithelial to mesenchymal conversion. Breast Cancer Res. 3, 213-217 (2001).
15. Quante, M. et al. Bone marrow-derived myofibroblasts contribute to the mesenchymal stem cell niche and promote tumor growth. Cancer Cell 19, 257-272 (2011).

16. Yeh, H. W. et al. A new switch for TGFbeta in cancer. Cancer Res. 79, 3797-3805 (2019).

17. Mani, S. A. et al. The epithelial-mesenchymal transition generates cells with properties of stem cells. Cell 133, 704-715 (2008).

18. Ikushima, H. \& Miyazono, K. TGFbeta signalling: a complex web in cancer progression. Nat. Rev. Cancer 10, 415-424 (2010).

19. Mishra, P. J. et al. Carcinoma-associated fibroblast-like differentiation of human mesenchymal stem cells. Cancer Res. 68, 4331-4339 (2008).

20. Turner, N. \& Grose, R. Fibroblast growth factor signalling: from development to cancer. Nat. Rev. Cancer 10, 116-129 (2010).

21. Navid, S. et al. The fibroblast growth factor receptors in breast cancer: from oncogenesis to better treatments. Int. J. Mol. Sci. 21, 2011-2026 (2020).

22. Presta, M. et al. Fibroblast growth factors (FGFs) in cancer: FGF traps as a new therapeutic approach. Pharm. Ther. 179, 171-187 (2017).

23. Hanahan, D. \& Weinberg, R. A. Hallmarks of cancer: the next generation. Cell 144, 646-674 (2011)

24. Sahai, E. et al. A framework for advancing our understanding of cancerassociated fibroblasts. Nat. Rev. Cancer 20, 174-186 (2020).

25. Cirri, P. \& Chiarugi, P. Cancer-associated-fibroblasts and tumour cells: a diabolic liaison driving cancer progression. Cancer Metastasis Rev. 31, 195-208 (2012).

26. Toullec, A. et al. Oxidative stress promotes myofibroblast differentiation and tumour spreading. EMBO Mol. Med. 2, 211-230 (2010).

27. Zeisberg, E. M. et al. Discovery of endothelial to mesenchymal transition as a source for carcinoma-associated fibroblasts. Cancer Res. 67, 10123-10128 (2007).

28. Luo, J., Chen, X. Q. \& Li, P. The role of TGF-beta and its receptors in gastrointestinal cancers. Transl. Oncol. 12, 475-484 (2019).

29. David, C. J. \& Massague, J. Contextual determinants of TGFbeta action in development, immunity and cancer. Nat. Rev. Mol. Cell Biol. 19, 419-435 (2018).

30. Colak, S. \& Ten Dijke, P. Targeting TGF-beta signaling in cancer. Trends Cancer 3, 56-71 (2017).

31. Papageorgis, P. TGFbeta signaling in tumor initiation, epithelial-tomesenchymal transition, and metastasis. J. Oncol. 2015, 587193 (2015).

32. Zhou, Y. et al. FGF/FGFR signaling pathway involved resistance in various cancer types. J. Cancer 11, 2000-2007 (2020).

33. Hessmann, E. et al. Fibroblast drug scavenging increases intratumoural gemcitabine accumulation in murine pancreas cancer. Gut. 67, 497-507 (2018). 